Voix et Images

voixetimages

\title{
Au ban des lettres. Hors-texte et robe-prétexte
}

\section{Gabrielle Poulin}

Volume 5, numéro 2, hiver 1980

Yves Thériault

URI : https://id.erudit.org/iderudit/013885ar

DOI : https://doi.org/10.7202/013885ar

Aller au sommaire du numéro

\section{Éditeur(s)}

Les Presses de l'Université du Québec

\section{ISSN}

0318-9201 (imprimé)

1705-933X (numérique)

Découvrir la revue

\section{Citer cet article}

Poulin, G. (1980). Au ban des lettres. Hors-texte et robe-prétexte. Voix et Images, 5(2), 381-393. https://doi.org/10.7202/013885ar d'utilisation que vous pouvez consulter en ligne.

https://apropos.erudit.org/fr/usagers/politique-dutilisation/ 


\section{Au ban des leltres \\ Hors-texte el robe-prétexte}

D'un cỏté, le gauche plus précisément, s'étend un long couloir sans porte ni fenêtre. II n'est pas obscur cependant. La ligne bleue, rigide, qui le délimite à droite, laisse passer la lumière. Elle ne retient pas non plus les sons ni les odeurs. Mais une fois que vous vous étes laissé attirer dans cette galerie étroite, vous êtes enfermé à perpétuité. A moins que...

Au début, on ne prend pas garde à l'exiguitté du lieu. On est un peu enivré par l'altitude. Pour ma part, j'ai longtemps cru que les marges où $j$ 'avais établi domicile m'assuraient d'une position privilégiée. Bien sûr, j'évitais de regarder vers la gauche où tout me semblait informe et irréel comme dans les profondeurs d'un gouffre. Je n'aurais pas eu l'audace d'occuper le vaste espace de la page blanche qui s'étendail à ma droite, altirant comme un mirage, auquel devaient avoir accés les seuls élus de la littéralure. Le destin avait voulu que je sois attachée aux confins du réel et de l'imaginaire el que je demeure vigilante dans la zone vide des frontières.

\section{De quelques régles deflinles d'un pases Indelln!}

C'est dans les marges des cahiers d'écoliers que j'ai subi mon premier emprisonnement volontaire. Mais j'étais une captive heureuse. Je parcourais cet espace blanc comme une voie lactée, en allumant, ici et là, des étoiles bleues, vertes, rouges et dorées. Les $A$, les $O$ et les $E$, qui dansaient avec les $I$ et les $U$, entre les doubles barbelés des lignes, se mettaient à scintiller et j'essayais de lire, a travers leurs silhouettes solitaires ou leurs couples fragiles, les signes tremblants des prodiges qu'ils annoncaient. Puis, les marges se rétrécirent.

On ne s'imagine pas à quelles acrobaties doit s'adonner l'institutrice au cours d'une langue carrière. En ce temps-là, la maitresse d'école des champs, dès sa première année d'enseignement, devait gravir chaque jour les marches raides de ses sept divisions: monter, descendre, monter, descendre. Inlassablement. La maitresse de ville, elle, commençait sa carrière tout au bas de l'échelle. Si elle enseignait dans une école de garçons, elle restait toute sa vie sur le premier ou le deuxiéme échelon. C'etait pour le bien des petits, qui 
pouvaient ainsi s'abriter encore une couple d'années sous ce pan rajouté à la jupe maternelle; c'était pour son bien a elle, qui n'aurait pas eu le bras assez vigoureux pour tenir ferme les classes des grands. Sans compter ce qu'il y aurait eu d'inconvenant, voire de risqué, a confier des garçons de huitième ou de neuvième année à une maîtresse, jeune par surcroît. Il arrivait même que des directeurs d'écoles exilent les toul-petits dans les gynécées voisins et gardent ainsi leurs murs à l'abri des vents de la séduction.

Dans les écoles de filles, presque toutes dirigées par les religieuses, la première année était sacrée: c'était celle de la première Communion. On n'aurait jamais abandonné le soin des futurs communiants à une laïque, ni non plus à une commençante. II n'était pas rare de voir, à la tête de ces classes, de vieilles religieuses qui avaient consacré leur carrière à l'enseignement des petits. Je me souviens de l'une d'entre elles qui était toute menue et avait encore, a cinquante ans, le visage lisse et blanc d'une premiere communiante. N'eút été la gravité un peu lourde de son habit religieux. elle aurait pu aisement etre confondue avec les petits qui voltigeaient, jasaient et picoraient autour de sa pelerine et de son voile, toujours posés un peu de guingois sur une forme trop fragile et trop familière pour qu'elle puisse jouer dignement le róle d'épouvantail. S'il y avait des professeurs laïques, bien entendu c'étaient des fermmes et, en général, elles enseignaient en deuxième et en troisième année. Les religieuses, elles. pouvaient s'attendre, même si leur ascension était plus irrégulière et plus lente que celle d'une écolière normale, de gravir, avec les années, les échelons du cours primaire tout au moins.

Pour moi, après avoir joué le róle de tée laïque des étoiles quotidiennes auprès de quarante-quatre petits garçons de deuxième année, à l'école SaintJean-de-Brébeuf, j'ai dû apprendre chez les écolières le maniement de plus en plus perfectionné et difficile du crayon rouge. A l'École normale, on avait essayé de nous inculquer, entre autres principes pédagogiques, celui-ci, qu'il vaut mieux récompenser que punir, relever les qualités que souligner les délauts. Allez donc obéir aveuglément é cet axiome quand il vous faut, chaque jour, corriger trente-cinq dictées d'élèves de cinquième ou de septième année. Hélas! je dois le confesser, j'ai pourchassé avec un plaisir douteux les petites bêtes qui font tant de ravages dans les longues chevelures des verbes et des participes français. Je les ai attirées dans le piège toujours ouvert des marges blanches sur lesquelles elles laissaient des traces de sang tandis que le front de l'enfant brûlait, " plein de rouges tourmentes".

Heureusement, ce crayon jouait son rôle avec plus de noblesse dans le cahier de compositions. Parce que ce sacro-saint cahier avait procuré ma propre adolescence studieuse ses plus grands soucis et ses plus grandes joies, je me suis toujours imaginé qu'il était le creuset dans lequel je pourrais voir s'accomplir les prodiges que l'enseignement de l'orthographe, de la grammaire, de l'analyse logique et de l'explication de texte avait pour mission unique de préparer. Avais-je le sentiment de participer à la poursuite et à la conquête d'une terre promise quand, toujours retenue dans la marge, je 
contemplais ces paysages lointains qui me seraient à jamais inaccessibles et traçais d'immenses " B* de triomphe à l'aide du bảton miraculeux qui, me l'imaginais-je, avait la vertu de färe jaillir l'inspiration et d'étancher les soifs futures? II m'arrivait souvent de remplir l'espace étroit qui m'était réservé de suggestions, d'interrogations, de points d'exclamation rutilants. Je consacrais des fins de semaine entière à ce travail que je croyais indispensable au progrès de mes élèves, m'imaginant volontiers que leur succès et leur bonheur dans la vie dépendaient de leur maitrise de la langue française (oui, oui, de l'assimilation également des leçons de catéchisme!), et que de grandes consolations leur seraient procurées par la lecture el par l'écriture. Le lundi matin, quand je leur avais remis leurs cahiers, apres avoir, comme il se doit, procédé une longue critique orale et avoir lu les meilleures compositions a haute voix, j'étais toujours douloureusement surprise de constater avec quelle rapidité, voire quelle négligence, mes élèves lisaient ou faisaient mine de lire, resserrés dans les marges, les signes rouges qui disaient, en phrases forcément elliptiques, la beauté ou l'originalité de leurs textes. C'est toute ma fin de semaine qu'elles rayaient d'un geste en referman trop vite leurs cahiers. II ne me restait plus à moi qu'un sentiment de lassitude et, dans les mains, que ce crayon rouge usé. Je me mettais à redouter l'arrivée du prochain samedi : les marges où je persistais à m'enfermer menaçaient de s'étrangler. Pour ranimer ma ferveur, je me procurais au plus tôt un nouveau crayon rouge sur lequel je m'appuyais et auquel je m'accrochais pour faire mon devoir et continuer accuper le seul espace - on! combien aride et désert! - qui m'était ménagé dans l'univers de la littérature.

\section{De quelques libertes imparfalies d'un passe antérieur}

La tittérature! A l'époque où j'étais encore une écolière et fréquentais l'école Marie-Immaculée, sise sur la rue Marie-Anne entre les rues Garnier et Delanaudière, nous ne laisions pas d'études de lettres. Seulement, chaque vendredi soir, a quatre heures, nous emportions dans notre sac d'école, soit notre cahier de compositions, qui était un beau cahier à l'encre plus épais que les autres, soit notre cahier à anneaux, dans lequel nous rangions les fouilles volantes de nos journaux personnels. Même si nous n'avions aucune idée de ce qui devait bien s'appeler, même en ce temps-là, les genres littéraires, nous savions très bien qu'une fin de semaine sur deux nous devions raconter une histoire, que nous pouvions inventer de toutes pièces, ou decrire un paysage, un objet, un animal, voire un personnage, que nul n'était obligé de reconnaître: c'était la narration, la description ou le portrait. Aucune restriction quant a la longueur du texte. Mais, gare aux fautes d'orthographe ou de grammaire qui menaçaient de leurs plaies hideuses l'integrité, la beauté el la valeur en pourcentage des plus purs chefs-d'ceuvre même! (Avons-nous assez pleuré sur les régles d'accord de "mêmen, " tout "et "quelque ", que nous croyions ne devoir jamais employer dans la vie, quelles que fussent les circonstances !) L'autre fin de semaine était consacrée au journal personnel. La prose de cet exercice devait être contenue tout entiere sur une feuille, recto et verso, que nous remettions, a la dérobée, sur le pupitre de la 
maitresse, en arrivant en classe le lundi matin. Je me souviens que je m'en retournais a ma place en rougissant de confusion et de plaisir, convaincue que la religieuse était déja en train de lire la première page qui révélait certains aspects réels de ma vie, un peu retouchés il est vrai, étrangers a la vie de l'école.

Voici, soudain que je ressens de nouveau la même impression. Quelqu'un n'est-il pas en train de lire en arriere de moi, tandis qu'il ne reste, penchée sur ma page blanche, que l'ombre de ces épaules et de ces mains qui ne sont déjá plus les miennes? Pour ce lecteur que n'auraient pas rejoint les ceuvres éphémères d'un mouvement littéraire de jeunesse qui connut un rayonnement parallele aux mouvements d'Action catholique, je m'en vais tenter une expérience de résurrection:

Petite illustration apocryphe d'un genre littéraire extermine sous les coups rouges des crayons des fonctionnaires tranquilles d'une antique révolution

Elles s'appelaient scour Pauline-Francoise, scour Émile-de-Jésus ot scour Louise-Estelle. Maintenant que les religieuses ont repris teur nom de fille, laissé repousser et libéré les boucles de lour chevelure, les noms que l'Évêque lour avait imposés, avec le voile, le jour de la véture, appartiennent au registre de l'imaginaire. Sans doute ne reconnaitraisje pas mes maitresses de septieme, huitieme el dixième annéo de l'école Maríe-Immaculéo, s'il m'arrivait de les rencontrer au hasard des rues dans lesquelles elles circulent, désormais libres, inconnues et ordinaires. (Le visage de mon professeur de neuvieme anné fait partie, lui, de mes images de cauchemars. II m'arrive encore de voir sceur X, la nuit. Elle ressemblait à un chat volant et f'ombre griffue qu'elle projetait sur mes cahiers les lacerait.) A ces trois femmes, qu'on doit confondre avec des grands-meres aujourd'hui, ie souhaite seulement, si elles lisent cette page de journal personnel, qu'elles reconnaissent les noms qu'elles portaient dans une vie antérieure. Des générations d'adolescentes montréalaises, j'en suis sûre, les ont retenus. Moi, qui ne lour en connais pas d'autres, io les conserve preciousement et il m'arrive de puiser dans leur évocation une sorte de puissance incantatoire. PaulineFrancoise, Emile-de-Jesus, Louise-Estellol Des anges passent sur la blancheur de mes pages et, comme autrefois, miraculeusement, sous leur habit noir, uniforme of luisant d'usure, les mots se mettent a respirer. "La cime, disaient-elles, a laissé tomber son petit chapeau dans l'abime. "Quand i'ai vu emerger trois crëtes blanches, eclatantes, de la blancheur des nuages, j'ai serré le bras de mon mari, comme jo fais toujours quand je veux attirer son attention. II s'est penche devant moi, vers le hublot et, dans son oreille toute proche, j'ai murmure, en les scandant comme des vers, les mots de cette regle d'orthographe, surgie d'une terre préhistorique: " La cime a laissé tomber son petit chapeau dans l'abîme. "Il a cru que c'était encore une de mes comptines. Les trois petites tétes blanches, rassurantes, n'ont pas bronché. Le ciel, audessus, etait d'un bleu impassible et, dans ma mémoire, je voyais s'engoutfrer les cornettes et les voiles noirs. Mon mari a serré ma main ot le vertige s'est dissipe. J'ai su que les trois crêtes brilleraient toujours dans la splendeur de leur neige éternelle. Je m'en doutais dejà quend, 
sur mes feuilles volantes, de mon écriture appliquée d'écoliére, je couvrais le mot "cime" d'un point lumineux, tandis qu'il lallait, c'était l'époque! que l'accent soit mis sur tant d'abimes.

J'ai été lente à comprendre que la pratique scolaire du journal personnel différait grandement de celle du vrai journal - du journal vrai ? - que je rédigeais tous les soirs avant de m'endormir, que je cachais dans un tiroir de ma commode, sous une pile de linge, ef dont je gardais la clef suspendue à une chaînette à mon cou, entre ma médaille miraculeuse et ma médaille scapulaire. A la longue, j'en suis quand méme arrivée à ma propre conception du journal personnel qui, pour moi, ne se distinguait plus de la composition que par une seule caractéristique - mais de taille! - : le choix du sujet en était entièrement libre. L'emploi de la première personne, il va sans dire, était de rigueur, mais ne constituait pas vraiment une difficulté. Au contraire. On aurait dit que le recours au aje" permettait d'accéder plus vite á l'univers merveilleux de l'invention."

Cette pratique du journal personnel, je crois, avait cours dans toutes les écoles de la CECM et n'avait pas été inventée par les religieuses. comme semble le croire Thérése, l'héroïne-narratrice d'Une forét pour Zoe, qui a conservé un souvenir bien différent de l'exercice de ce genre littéraire:

Marie se vengeait de la vie joyeusement en se moquant de nos maitresses, a leur insu, dans le journal qu'elles nous commandaient tous les lundis. Sous le couvert d'un essai de littérature, ce journal n'avait d'autre but que de les renseigner sur la qualité de l'atmosphère familiale et sur notre emploi du temps dans les lieux profanes, hors de leur surveillance. (Louise Maheux-Forcier, Une torét pour Zoé, CLF, 1969, p. 173.)

La plupart des romanciers québécois actuels, du moins ceux d'un certain áge, qui disent "je» et ont toujours l'air de raconter des expériences vécues, surtout quand ils les inventent, ont dû se plier à l'exercice du journal personnel, qui était la forme mondaine, articulée et glorieuse de l'autre exercice, secret, bredouillé et honteux, la confession, à laquelle ils devaient se soumettre chaque samedi soir mêmement. L'écolier des années 40 se purifiait des humiliations subies derrière la grille en s'enveloppant de la page blanche, véritable robeprétexte, qui lui permèttait d'entrer dans la vraie vie où l'innocence et la passion se concilient el s'appellent. (Quant à la pratique actuelle, qui s'est imposée aux critiques orthodoxes, de l'application de grilles entre la page écrite et l'cil lucide et froid - combien de fois? comment? Non! Non! le pourquoi est inutile et sans intéret -, il serait sans doute abusil et farfelu de lui chercher des antécédents dans la liturgie du sacrement de l'Absolution.

Pour moi, la page blanche, chaque semaine, s'ouvrait comme un portail et ce «je”, écrit sur la première ligne, jouait le rōle d'une clef magique. Pour

- Longue note infrapaginale qui résiste au renvoi dans la marge la plus basse do la page. 
être bien sùre de demeurer dans les limites du journal personnel, j’avais soin de choisir un point de départ réel: une promenade dans le parc Lafontaine, par exemple, le dimanche après-midi. Toute la classe, me disais-je, pourra reconnaître le court de tennis, le kiosque a musique, la fanfare, les allées ombragées avec leurs bancs verts, l'étang des canards, la fontaine lumineuse et, à l'autre bout tout à fait, bien au-delà du petit pont romantique (que de photos mes tantes, en promenade dans la Métropole, ont prises de la ribambelle de cousins que nous étions sur, et sous, ce pont-jouet!), l'édifice imposant de la bibliotheque municipale où je me rendais réellement tous les samedis pour échanger mes trois livres (au sous-sol, bien sür! II fallait avoir seize ans pour obtenir l'autorisation de s'abonner à la bibliothèque des adultes. J'allais quand même me réfugier dans la salle de lecture de la vraie bibliothèque pour rédiger ma composition ou mon journal personnel). Je choisissais donc l'un ou l'autre de ces points de repère qui devenaient à tour de rôle des tremplins pour les danses et les sauts de l'imagination. Non, je n'avais jamais lu le Paysan de Paris ni Nadja et je ne savais pas qu'un dénommé André Breton était à la veille de faire escale au milieu des fous de Bassan à l'lle Bonaventure. A ma façon, je cherchais à découvrir, dans ce lieu privilégié, hanté par tous les amoureux du Plateau Mont-Royal, les signaux qui annonçaient la présence de l'insolite. les rencontres merveilleuses et déclenchaient reconnaissances, métamorphoses et. pour tout dire, a l'amour fou". Les lectures qui nous étaient permises pourtant étaient aux antipodes du surréalisme. Comme toutes les adolescentes de mon milieu, j'étais renfermée dans la a Bibliothèque de Suzette * dont les livres aux couvertures bleu poudre évoquaient les nostalgiques salons de la comtesse de Ségur où nous étions invitées à contempler des petites filles à crinoline, avec de longs bas blancs et des escarpins vernis, orphelines ou enfants trouvées, pleurant dans leurs longs cheveux d'or et leurs rubans de velours, avant de retrouver ou de " reconnaitre" miraculeusement leurs merveilleux et nobles parents. Je ne devais être arrachée à la divine comtesse (en réalité, d'elle, comme aurait dit Emma Rouault, j'avais tout lu!) que pour ètre contiée au rassurant René Bazin, à ce digne Paul Féval, à ce saint Pierre l'Ermite et aux plus édifiants de nos auteurs québécois: Michelle Le Normand, Marie-Claire Daveluy et Léopold Desrosiers.... Oui, je le confesse, j'ai aussi pleuré dans la Maison des rossignols et, je crois bien. dans tous les romans de Delly, jusqu'au jour ou I'on m'a fait découvrir les Brigitte: Brigitte jeune fille, Brigitte jeune femme, Brigitte maman, que j'ai lus et relus dans l'attente des tomes à venir. Dans l'altente également des vrais livres qui devaient bien exister quelque part...

Assez curieusement, mon jeune frère semblait connaitre des livres bien différents des miens. La rougeur m'était montee au front, sans que je sache exactement pour quelles raisons obscures, le jour où, ayant ouvert en cachette l'un de ces livres, i'y avais lu un passage ètrange:

Petit résumé traditionnel et désuel d'un texte dépouillé de toute attache et délesté de toute référence.

En posant les mains sur la jeune fille qu'il avait invitèe dans sa couche, le roi avait eu la surprise et le plaisir de constater qu'elle avait revêtu une 
longue jaquette (je pense bien que l'auteur, sans que nul reprèsentant de l'Académie n'ait eu à le censurer, avait dú écrire " chemise de nuit "), qui s'ouvrait sur le devant comme la porte d'une " armoire aux sortilèges". L'ingénue avait alors confié a son royal amant que c'était par respect pour lui qu'elle s'était fait coudre cette jaquette - pardon, Majesté! - cette chemise de nuit extraordinaire. N'eūt-il pas été inconvenant, en effet, que le roi, force de retrousser (non, c'est inutile! "chemise de nuit" fait trop sophistiqué pour qui revient toujours au galop du naturel!) une jaquette conventionnelle fût contraint d'enfouir ses narines augustes dans celte partie du vêtement qui avait d'abord touché les pieds de la jeune vierge?

J'avais détourné pudiquement les yeux de cette armoire interdite et refermé le livre lentement, avec un peu de regret. Tout cela était raconté avec beaucoup de légèreté et une sorte d'humour qui, bien que je le ressentisse, ne me faisait pas sourire comme si l'ignorance et le scrupule avaient posé leur voile sur mes yeux et sur mon esprit. Il faudra de longues années avant que j'ose moi aussi découdre ce voile de haut en bas.

Mon frère lisait également des poètes. Je l'entendais parfois réciter des vers étranges, quand ses amis du collège Sainte-Marie (l'un d'eux s'appelait déjà André Belleau) venaient à la maison:

Mais, vrai, j'ai trop pleuré! Les Aubes sont navrantes

$O$ que ma quille éclate! $O$ que j'aille à la mer!

Je suis gai! Je suis gai! Vive le soir de mai!

Mon ange, ma sceur

Songe à la douceur

D'aller là-bas vivre ensemble

Que ton vers soit la bonne aventure

Eparse au vent crispé du matin

Qui va fleurant la menthe et le thym

Et tout le reste...

Je me surprenais a écouter les voix muantes de ces adolescents, qui se cachaient pour fumer leur première cigarette et s'enivraient de ce vin âpre d'une poésie d'où ne s'échappail, pour me rejoindre dans ma chambre, qu'un bouquet grisant. Tout à coup, mes livres me paraissaient fades. Je fermais les yeux et je murmurais comme on récite une formule magique:

Sur l'onde calme et noire où dorment les étoiles

La blanche Ophélia flotte comme un grand lys

Je ne savais plus comment disposer les a longs voiles" de ces vers dont me parvenait encore de la chambre prochaine la douceur des "hallalis". Aucun des livres que j'avais lus n'avait exercé sur moi cette fascination. Non. il n'y avait pas de "hallalis" dans les livres recommandés pour les adolescentes. Les petites Québécoises des écoles de la CECM. puis plus tard, des Ecoles normales, apprenaient la lecture et l'écriture à l'abri des textes, en marge de la vie et des lettres. Elles savaient faire une dictée sans faute, analyser une phrase bourrée de propositions subordonnées: relatives, subjonctives et 
circonstancielles de temps, de cause, de condition, de but, de concession, d'addition, de restriction, de conséquence, de comparaison... ; elles pouvaient composer une narration impeccable avec une introduction, un nceud et un dénouement, faire le portrail de leur petite amie de ccour ou de leur chat (non, non, la plupart ne connaittraient jamais Colette!), décrire la chute des feuilles en s'inspirant d'Albert Lozeau:

Dans le vent qui les tord les érables se plaignent,

Et j'en sais un, lâ-bas. dont tous les rameaux saignent!

el un paysage embelli par la première neige, paisible et rassurant comme un poème de Chauveau:

On! que j'aime la neige! Oh! que j'aime a la voir

Descendre par flocons sur le sol encor noirt

Ou bien, quand elle tombe en poussiere si fine

Que l'on croirait qu'un ange épand de la farine

Pour donner des gâteaux à nous, petits enfants.

Mais, jamais, au grand jamais, on n'entendait de hallalis entre les lignes droites de leurs cahiers impeccables. Les petites filles et les grandes filles modèles écrivaient, noir sur blanc, dans l'étroitesse et le silence de ce lieu où les maintenaient leurs mères et leurs institutrices, gardiennes de la tradition. landis que, dans les vastes espaces blancs où étaient appelés leurs fréres, les voyelles eltes-mêmes étincelaient: " I » rouge, " $U$ * vert, et dans l' $\alpha$ o bleu, l'on percevait les " strideurs étranges ", " silences traversés des Mondes et des Anges".

\section{A la recherche du plus-que-parfalt}

Le monde. Les Anges. Le combat était inégal. Aux poètes et à leurs mâles admirateurs, la louange de la mer et des champs, la maîtrise des villes, la folie des aventures, des voyages et la poursuite des amours! A nous, leurs sœurs douces et pures, la retraite humble, paisible et silencieuse du foyer ou du cloitre. Le culte de l'idéal n'avait rien à voir avec la culture des belleslettres. Lire? "Les romans sont comme les champignons: les meilleurs ne valent rien." (II semble qu'on ait changé d'avis ces dernières années, du moins à l'égard des champignons). Ecrire? Oui, mais à condition que votre plume vous emporte comme les ailes d'un ange vers les plus hauts sommets de l'idéal. Monsieur le Principal Bélanger, avec une onctueuse familiarité, appelait ses normaliennes, futures éducatrices : "les petites auxiliaires laïques du sacerdoce". De la "Vallée des champs" (nous n'avions pas attendu la loi 101 pour franciser le nom de Valleyfield, cette ville prédestinée oú croissaient, a l'abri des murs étanches d'un séminaire et d'une école normale, les fleurs mâles et les fleurs temelles qui devaient former la parure de l'Eglise de demain), on passait presque sans transition au jardin fermé de la montagne. "On n'allume pas une lampe pour la mettre sous le boisseau. "Les vierges sages entraient dans la salle des noces avec l'Époux. Ma lampe vacillante a étè suspendue avec les autres et je l'ai fixée dévotement tout au long des 
nuits, pendant... L'huile brúlait, mais le temps, lui, s'était arrêté. Il n'avait pas plus d'importance que l'espace. Une assoiffée d'absolu et d'éternel abandonne sans regrets ces concepts mondains aux laics et aux romanciers. Elle reste immobile, jeune toujours, impassible, dans l'attente calme et résignée de la récompense éternelle.

Petit à petit, "la vie dans le monde" a pris les apparences scabreuses d'un roman pour "adultes avertis". Bâtis dans les marges de la cité, les couvents se devaient d'exercer une fonction de vigilance assez semblable à celle de la censure sur les voix dont l'écho parfois leur parvenait, sur les mouvements qui arrivaient, sinon à les ebranler, du moins à les inquiéter. Entre la vie religieuse et la vie tout court, se dressaient les garde-fous de l'obéissance, de la chasteté et de la pauvreté et un nombre incroyable de règles barbelées, dressées et tirées au-dessus du précipice.

\section{- Mais que vous vous êtes éloignée de la littérature!}

Justement, voici que j'y reviens. Ou plutót quelqu'un m'y ramene qui, n'ayant jamais craint ni les géants ni les armées bardées de fer, avait osé me suivre aux marches du réel: s'il n'avait pas pu m'empècher d'entreprendre cette aventure démiurgique, il espérait pouvoir un jour me délivrer des chaines plus lourdes de jour en jour, dont je m'étais laissée charger.

Non, qu'on se rassure! Je ne croyais plus aux contes de fées et j'avais dû renoncer à Delly et à tous ses avatars. Certes, j'avais gardé la passion des livres, mais, sur les rayons des bibliotheques de nos couvents, on ne trouvait, en ce temps-là, que des vies de saints. Aussi ai-je dû me passionner, un temps du moins, pour l'hagiographie. Sainte Jeanne de Chantal, saint François de Sales, sainte Thérèse d'Avila, saint Jean de la Croix, Élisabeth de la Trinité (saint Augustin était introuvable), saint Vincent de Paul et tant d'autres, sont devenus, à tour de róle, les modèles d'une vie intérieure polymorphe.

\section{Les tentations du conditionnel ef les pleges de l'irrét}

Les supérieures auraient dû se défier de ces lectures intempestives. A qui le venin de la métamorphose a été inoculé dés le berceau, tous les livres sont nocifs. Au * vestibule du paradis ", les vies de saints, prenant la relève des romans de la bibliothéque rose ou bleue, me tenaient lieu de aparadis artificiels". Sur le seuil, mon chevalier, lui. veillait et attendait. Si j'appelle Don Quichotte, mon chevalier, ce n'est pas pour l'asservir à une tâche indigne de lui et de ses sublimes ambitions. Non, je dis mon chevalier, comme je dirais mon parrain. J'ai longtemps cru que deux anges étaient postés d'office auprès du berceau des chrétiens: le bon ange, a droite (le petit Québécois, a l'aide de cette référence sacrée, apprenait très tót à distinguer sa main droite, celle qui devait faire le signe de la Croix, de sa main gauche, celle qui ne devait pas apprendre á écrire) : á gauche, le mauvais. Bientôt, sur la foi des contes de mon grand-père, je me suis mise à imaginer qu'il y avait eu aussi des fées et des sorcières tout autour de mon berceau. Vers l'âge de douze ans, je me suis mème bercée de l'illusion que, peut-étre, il y avait eu des 
Muses. Maintenant tout ce cortège s'est dissipé. II ne reste, penchée sur les rêves fragiles de la petite fille de jadis, que l'ombre gigantesque, projetée sur le mur de la cuisine, de la chaise berceuse dans laquelle, tous les soirs, mon père, entourant mon corps de son bras gauche, miendormait, en lisant tantôt silencieusement, tantôt á voix haute, et laissait parfois éclater son rire qui faisait bouger les ombres sur les murs. Du petit livre qu'il tenait dans sa main droite, s'échappait en piaffant le cheval de Don Quichotte. Bientôt, nous partions dans une chevauchée folle autour de la cuisine. Les ombres se métamorphosaient, se mettaient à vivre: la chaise de bois caracolait. Quand, de sa main gauche, mon père tournait la page, il devait resserrer son étreinte. Sur le mur, on voyait tourner les ailes des moulins, gambader des jeunes moutons, se dessiner des forteresses, des cháteaux, s'abaisser des pontslevis. Bientót l'ombre du cheval et du chevalier remplissait toute la piéce: derrière les paupières closes de la petite fille se levaient des lueurs étranges, des terres inconnues. Les craquements de la chaise berçante s'enflaient comme le bruit des torrents. L'univers était trop grand tout à coup; les montagnes, trop hautes; les foréts, trop denses. Mais, voici que les parois des rochers renvoyaient soudain les échos d'un rire rassurant. Le cheval allait au pas, maintenant. Le Chevalier-de-la-triste-figure se mettait a sourire. Tout devenait immobile et blanc: la vie et la mort n'avaient plus qu'un visage sans ombre. Entre les pages du livre abandonné. seul un signet pâle laissait présager, sur l'obscurité des signes, le retour de la vie fragile dans la lumière.

Si j'ouvre le petit livre jauni, aux caracteres à demi effacés, à la page exacte où brille le signet de soie ancienne brodé de fleurs roses et de guirlandes dorées, je lis cette phrase du Quichotte qui contient le secret qu'il a jadis murmuré tant de fois à mon oreille comme une formule magique:

[...] dans l'univers il n'est qu'un seul bien digne des efforts, des travaux, de l'amour des hommes: ce bien, c'est la liberté! Tous les trésors qu'enferme la terre, tous ceux que possède la mer, toutes les jouissances que promet la fortune, tous les plaisirs qu'inventa la mollesse, ne peuvent étre comparés à cette liberté précieuse pour laquelle le mortel qui pense expose sans cesse ses jours et les sacrifie avec joie.

Donc, ayant enjoint à Rossinante de l'attendre à la barrière, mon parrain, brandissant son épée, s'est frayé un chemin parmi les ombres. Il avait l'air si respectable et si digne dans son intrépidité, que personne ne lui a opposé de résistance. II est venu jusqu'à la porte de ma cellule qui s'est ouverte d'ellemême dans un grand vent de folie et de liberté.

Sur le coup, je n'ai pas reconnu le chevalier de mon enfance. D'ailleurs, il était déjà reparti, appelé vers le nord de la cité, où, comme je l'ai appris plus tard, l'attendait une autre aventure de "démanche».

\section{Les "marges blanches" du futur}

Avant d'entreprendre, enfin! ma propre chevauchée dans l'étendue des pages glacées, sur lesquelles chacun des pas creuse une empreinte blanche 
qui ne s'efface jamais, il me fallait encore être très sage: j'avais tout a apprendre. On ne s'échappe pas ainsi des corniches étroites et silencieuses qui bordent l'univers des hommes de tous les cótés des précipices, sans se croire appelé à d'autres fonctions de vigilance. Le destin avait voulu que je vive toute ma vie en marge du réel; quand, aprés avoir abandonné aux orties la robe aux plis rigides de la statue, je suis arrivée, comme une revenante, dans l'univers de la littérature, le hasard, celui qu'on appelle objectif, m'a jetée dans les " marges blanches" des rencontres fortuites et des reconnaissances merveilleuses d'un univers surréel qui s'appelait L'Amour la poésie:

Ses yeux sont des tours de lumière

Sous le front de sa nudité

Adieu voiles, jaquettes, chemises de nuit!

Les halos de la lumière

Aux lévres de l'horizon

J'avançais, j'avançais à tâtons :

Les ombres que tu crées n'ont pas droit a la nuit.

Voici de nouveau la barre rassurante sur laquelle je m'appuie. L'amour me ramène, moi qui avais été exilée de la littérature québécoise avant méme de la connaitre, aux marches poétiques du Pays. Je dessine mes propres appels et mes propres silences au bord des lles de la nuit, autour du Tombeau des rois. Je troque le crayon rouge des interdictions pour le noir qui peut, lui, inventer des ombres. Jacques Brault, Fernand Ouellette, Gitles Hénault offrent de grandes marges blanches à ma danse fraternelle. Je fais l'apprentissage du regard, de la parole et du geste. Me voici devenue image et rythme et démesure! Mes yeux s'agrandissent, mon oreille s'aftine. Tant et tant de parfums nouveaux m'enivrent. Mon crayon se met a poursuivre ses propres signes avec désinvolture. Attention! Attention! N'avez-vous pas de méthode d'approche, mademe la lectrice? N'y a-t-il pas une grille que vous pourriez poser humblement et pudiquement entre le texte et votre visage si effrontement découvert? Non, j'ai renoncé a jamais à toute forme d'emprisonnement et je piaffe d'impatience devant la ligne invisible qui me retient encore en marge de la page blanche. J'entends des appels, je commence á découvrir des sentiers secrets qui ont l'air de me laire signe. Je jette des cailloux blancs sur mon chemin futur. Peu à peu, sur la page blanche que je hante encore comme un fantóme, je vois prendre lorme la fresque que brossent les romanciers québécois. J'ai peine à retenir mon crayon qui s'emballe à chaque appel, à chaque parfum, a chaque image surgis comme miracles avec les ombres mouvantes des déserteurs sur le pays de papier. Major, Beaulieu, Carrier. Ducharme... ont chaussé des bottes-de-sept-lieues pour parcourir l'espace et le temps qu'ils inventent. Non, je ne revêtirai pas la blouse aseptique du chercheur formaliste pour étudier sans danger le fonctionnement des textes. J'ai follement envie, moi, d'être contaminée. La littérature québécoise s'ouvre devant mes yeux, jusquiici dangereusement préservés, comme un univers 
magique. Je ne sais pas distinguer le pays réel ni la vie vécue, de l'aventure de ces romanciers el de ces poètes que je ne connais pas autrement que par les visages qu'ils ont le pouvoir de se donner quand ils écrivent. Le temps et l'espace réels sont dèjoués. Nul héros ne s'abandonne plus aux règles bien définies du progrès historique. C'est partout la grande fête, le bal masqué, mais tout le monde se connait. Les personnages passent d'un livre à l'autre, des mains d'un romancier dans celles d'un autre. Ils révent, ils délirent. IIs se prennent pour leur propre créateur. Ils disent " je * et se croient " autre *; ils disent "il " et se retrouvent. Ils écrivent comme on respire, comme on vit. Je m'attarde au bord des pages. Tous ces personnages se superposent: ils forment un être hallucinant que je fixe, une sorte de béte immense qui posséde, me semble-t-il, le secret de la vie.

Le jour où j'ai vu la mer pour la première fois, - c'était a Nice, juste au pied de la Promenade des Anglais - je me suis mise à rire et à danser. Le soir était venu. Un gardien de chantier dont nous n'avions pas compris la mise en garde a lâché son chien sur moi. J'ai couru vers la rive, mais te berger allemand m'a rejointe avant que j'aie réussi à atteindre la mer. Depuis, j'ai peur de tous ces êtres qui aboient, qui vocifèrent et mordent pour empêcher les vivants de saluer et d'embrasser la mer quand ils la reconnaissent et pour les ramener, dociles piétons, sur les promenades policées, où une ligne bleue est tendue comme une barrière au-dessus du gouffre de l'imaginaire.

\section{Pour un présent omnitemporel}

Une brèche. Oui, pratiquer une brèche. Je me suis avancée de plus en plus près de la page hallucinante dans sa blancheur. Je me disais qu'un jour, j'aurais le courage d'enjamber le garde-fou, de me jeter dans le vide. Alors, je me suis penchèe tellement que, tout à coup. j'ai perdu, avec le sentiment rassurant de l'équilibre. la conscience du danger. J'aurais voulu d'emblée atteindre le fond des eaux, interroger les formes obscures des vivants qui se cachent au milieu des morts, revenir de temps en temps à la surface pour sentir sur ma peau troide la chaleur du soleil, la caresse de l'onde qui ne nous atteint plus quand nous sommes complétement immergés. La page blanche ne se laisse pas traverser si aisement. Elle offre aux regards de celui qui l'aborde pour la première fois la résistance séduisante de ses reflets. Tout apparaît étrange alors et familier. Le silence se fait autour de soi jusqu'á ce que, des images renversées, montent des voix inconnues. Le regard fasciné devient pour l'oreille et pour la main le grand pourvoyeur. Mais d'oú viennent ces images? II n'y a rien devant moi qui ressemble au monde réel qui m'entoure. D'ailleurs, je ne peux déjá plus jeter un regard circulaire dans ce présent qui devrait être le mien et je ne vois pas non plus devant moi mon propre reflet. Peut-ètre quelqu'un. sur la rive opposée, aperçoit-il, au milieu de ces formes renversées, le mouvement d'une silhouette sombre et fragile qui a l'air de marcher la téte en bas. Ce n'est pas moi non plus. Oú suis-je? Que suis-je devenue? Désormais, quand j'arrive au bord de la rivière blanche, je m'abandonne totalement à ce moment de la rupture de l'équilibre: je ne 
suis plus attachée au sol; je ne peux ni nager, ni voler, mais je fais de grands gestes fous qui font apparaitre des formes, des parfums et des couleurs nouvelles devant moi. Alors, je sais que je vis, même si le temps s'est arrèté: ici et maintenant, tout le passé des marges peut s'animer. Le crayon rouge ne dit plus: attention! stop! ni même arrết! II chante la vie et le sang des ombres que ma main trace el auxquelles la page blanche comme une grande robe d'innocence donne le pouvoir de vivre, de pleurer et de rire. Enfin!

La rivière blanche? Je ne saurai m'en détacher. Elle est plus puissante que les reflets qu'elle engendre et elle saura bien les emporter, comme des jouets arrachés par la débâcle printanière, jusqu'à la mer blanche. Le passé? Non, il n'appartient plus aux ombres. Le passé est un mot que l'écriture dépouille de tous ses vêtements. Il est nu comme l'enfant qui vient de naitre. comme la jeune fille qui s'offre à l'amour pour la première fois. Il danse, il chante, il pleure, il rit. Le présent danse et chante et pleure et rit avec lui. Sur la page blanche, on voit le reflet unique d'un couple renversé dans l'amour, à la recherche des merveilles qui se cachent dans les profondeurs où certains vivants arrivent, parfois, quand ils ont enfin abandonné même leur reflet à la grâce de la rivière blanche.

Gabrielle POULIN

Le 13 juin 1979 